\title{
Residues in two homology blocks on the amino side of the tRNase Z His domain contribute unexpectedly to pre-tRNA $3^{\prime}$ end processing
}

\author{
NEELA ZAREEN, ${ }^{1}$ ANGELA HOPKINSON, and LOUIS LEVINGER \\ York College of The City University of New York, Jamaica, New York 11451, USA
}

\begin{abstract}
tRNase Z, which can endonucleolytically remove pre-tRNA 3'-end trailers, possesses the signature His domain $(\mathrm{HxHxDH}$; Motif II) of the $\beta$-lactamase family of metal-dependent hydrolases. Motif II combines with Motifs III-V on its carboxy side to coordinate two divalent metal ions, constituting the catalytic core. The PxKxRN loop and Motif I on the amino side of Motif II have been suggested to modulate tRNase $Z$ activity, including the anti-determinant effect of CCA in mature tRNA. Ala walks through these two homology blocks reveal residues in which the substitutions unexpectedly reduce catalytic efficiency. While substitutions in Motif II can drastically affect $k_{\text {cat }}$ without affecting $k_{M}$, five- to 15 -fold increases in $k_{M}$ are observed with substitutions in several conserved residues in the PxKxRN loop and Motif $\mathrm{I}$. These increases in $\mathrm{k}_{M}$ suggest a model for substrate binding. Expressed tRNase Z processes mature tRNA with CCA at the $3^{\prime}$ end $\sim 80$ times less efficiently than a pre-tRNA possessing natural sequence of the $3^{\prime}$-end trailer, due to reduced $k_{\text {cat }}$ with no effect on $k_{M}$, showing the CCA anti-determinant to be a characteristic of this enzyme.
\end{abstract}

Keywords: pre-tRNA; 3' processing endonuclease; tRNase Z; homology blocks

\section{INTRODUCTION}

Transfer RNAs are central to protein synthesis. All tRNAs are transcribed as precursors and processed by removal of extra sequences from their ends before they can be aminoacylated and used in translation. The $5^{\prime}$-end leader is removed by a widely conserved enzyme, RNase $\mathrm{P}$ (for review, see Xiao et al. 2002). All mature tRNAs have CCA at the $3^{\prime}$ end, and there are two different paths to this end (for review, see Mörl and Marchfelder 2001). In Escherichia coli, CCA is transcriptionally encoded, and the $3^{\prime}$-end trailer is first cut by the endonuclease RNase E and then trimmed by a chorus of exonucleases ( $\mathrm{Li}$ and Deutscher 1996, 2002; Ow and Kushner 2002). In this organism, the CCA-adding enzyme is nonessential; the slow growth phenotype of the knockout suggests that it is

${ }^{1}$ Present address: Department of Biology, Fairchild Center, Columbia University, New York, NY 10027, USA.

Reprint requests to: Louis Levinger, Department of Natural Sciences/ Biology, York College of The City University of New York, 94-20 Guy R. Brewer Blvd., Jamaica, NY 11451, USA; e-mail: louie@york.cuny.edu; fax: (718) 262-2652.

Article published online ahead of print. Article and publication date are at http://www.rnajournal.org/cgi/doi/10.1261/rna.4206. important for repair of damaged tRNA 3' ends (Zhu and Deutscher 1987). In eukaryotes, archaea, and many bacteria in which CCA is not transcriptionally encoded-on the other hand-the CCA-adding enzyme is essential (Aebi et al. 1990). In these cases, in a widely conserved, efficient, and precise mechanism for pre-tRNA $3^{\prime}$ end maturation, a pre-tRNA $3^{\prime}$ end processing endonuclease, tRNase $Z$, cleaves the $3^{\prime}$-end trailer directly following the discriminator (the last unpaired nucleotide following the 7-bp acceptor stem), leaving a 3' $\mathrm{OH}$ to which CCA can be directly added (Castaño et al. 1985; Frendewey et al. 1985; Chen and Martin 1988; Marchfelder et al. 1990; Nashimoto 1992; Levinger et al. 1995; for reviews, see Levinger et al. 2004b; Vogel et al. 2005). Interestingly, tRNA precursors with and without transcriptionally embedded CCA coexist in Bacillus subtilis, and the two pathways for pre-tRNA 3' end maturation are used selectively on the appropriate substrates (Pellegrini et al. 2003). In this and other cases, CCA of mature tRNA is an anti-determinant that prevents mature tRNA from recycling through tRNase $\mathrm{Z}$ (Nashimoto 1997; Mohan et al. 1999). This has not been universally observed, however (Schiffer et al. 2003; see Discussion). In Thermotoga maritima, a bacterial species in which all but one of the pre-tRNAs have transcriptionally 
encoded CCA and important pre-tRNA $3^{\prime}$-end exonucleases are absent, tRNase $\mathrm{Z}$ cleaves atypically after CCA instead of after the discriminator (Minagawa et al. 2004).

tRNase $\mathrm{Z}$ is present in a short form (tRNase $Z^{S}$ ) in bacteria and archaea and in a long form $\left(\right.$ tRNase $\left.Z^{\mathrm{L}}\right)$ in eukaryotes (Schiffer et al. 2002). tRNase $Z^{\mathrm{L}}$ apparently evolved as a gene duplication of tRNase $Z^{S}$ in which the active site was retained in the carboxy half, leaving the amino half of tRNase $\mathrm{Z}^{\mathrm{L}}$ free to diverge (Tavtigian et al. 2001). For example, a flexible domain looped out from the globular core of tRNase $Z^{S}$ (de la Sierra-Gallay et al. 2005) that is involved in pre-tRNA binding (Schilling et al. 2005) was retained in the amino half and lost from the carboxy half of tRNase $\mathrm{Z}^{\mathrm{L}}$. Some eukaryotes (Saccharomyces cerevisiae, Caenorhabditis elegans, Drosophila melanogaster) have only tRNase $\mathrm{Z}^{\mathrm{L}}$, which, in the case of the fruit fly, is involved in both nuclear and mitochondrial pre-tRNA maturation (Dubrovsky et al. 2004), while some (e.g., Arabidopsis thaliana, Homo sapiens, and other mammals) have both tRNase $Z^{\mathrm{L}}$ and tRNase $Z^{\mathrm{S}}$.

Conserved homology blocks in the carboxy half of tRNase $Z^{\mathrm{L}}$ align with blocks in tRNase $Z^{\mathrm{S}}$. Motif II consists of the His domain $(\mathrm{HxHxDH})$, the signature sequence of the $\beta$-lactamase family of metal-dependent hydrolases, which, in collaboration with two other histidines and another aspartate (Motifs III-V), coordinates two divalent metals, constituting the active site. Two additional homology blocks on the amino side of the His domain (the PxKxRN loop and Motif I) have been suggested to modulate tRNase Z activity (Minagawa et al. 2004; de la Sierra-Gallay et al. 2005). Two residues in the Motif I region of $T$. maritima tRNase $\mathrm{Z}$ were suggested to be involved in its unusual selection of cleavage site and, by inference, the CCA anti-determinant (Minagawa et al. 2004). Alternatively, the PxKxRN loop could be involved in CCA anti-determination in B. subtilis tRNase Z (de la Sierra-Gallay et al. 2005); this loop is absent from T. maritima tRNase $\mathrm{Z}$ due to truncation of its amino end. To evaluate these hypotheses concerning regions and residues potentially involved in modulating tRNase $\mathrm{Z}$ activity, we analyzed the effects of Ala substitutions through the PxKxRN loop and Motif I of D. melanogaster tRNase $\mathrm{Z}$ on pre-tRNA ${ }^{\text {His }} 3^{\prime}$ end processing. Additionally, to quantitate the CCA anti-determinant effect, substrates possessing natural sequence of pre-tRNA and CCA following the discriminator were analyzed in parallel using expressed, affinity-purified tRNase Z.

\section{RESULTS}

\section{Homology blocks on the amino side of the His domain (Motif II)}

Multiple sequence alignments of tRNase $Z^{\mathrm{L}}$ and tRNase $Z^{\mathrm{S}}$ highlight the relationship of tRNase $\mathrm{Z}$ with other members of the $\beta$-lactamase family of metal-dependent hydrolases (Tavtigian et al. 2001; Schiffer et al. 2002; Takaku et al. 2003; Dubrovsky et al. 2004; Schilling et al. 2005) and the homology blocks most directly involved in catalysis (Motifs II-V in the terminology of Ishii et al. 2005). The PxKxRN loop and Motif I, two homology blocks on the amino side of Motif II (but still within the carboxy half of tRNase $Z^{\mathrm{L}}$ ) (Fig. $1 \mathrm{~A}, \mathrm{~B})$, are investigated herein, and the recently analyzed His domain (Motif II) (Fig. 1C; Zareen et al. 2005) is included to help establish context. Five eukaryotic tRNase $\mathrm{Z}^{\mathrm{L}} \mathrm{s}$ (above) and three bacterial tRNase $Z^{S}$ s (below) are shown in the alignments. Numbers above the top panels refer to the residues in fruit fly tRNase Z (a long form) (see Materials and Methods; Zareen et al. 2005) in which the central histidine of Motif II is H500 (Fig. 1C). Residue numbers and secondary structure elements directly above the tRNase $Z^{S}$ alignments refer to B. subtilis tRNase Z. The most conserved residues are in green, semiconserved residues are in red, and similar residues (e.g., large hydrophobics) are in blue. These homology blocks are observed in all species, except that T. maritima tRNase $\mathrm{Z}$ has no PxKxRN loop (Fig. 1A), as noted before (de la Sierra-Gallay et al. 2005).

\section{Correlation of homology blocks with the B. subtilis tRNase $Z$ structure model allows useful comparisons to be made between $t R N a s e Z^{L}$ and tRNase $Z^{S}$}

The structures of bacterial tRNase Zs (from B. subtilis, T. maritima, and E. coli) (de la Sierra-Gallay et al. 2005;

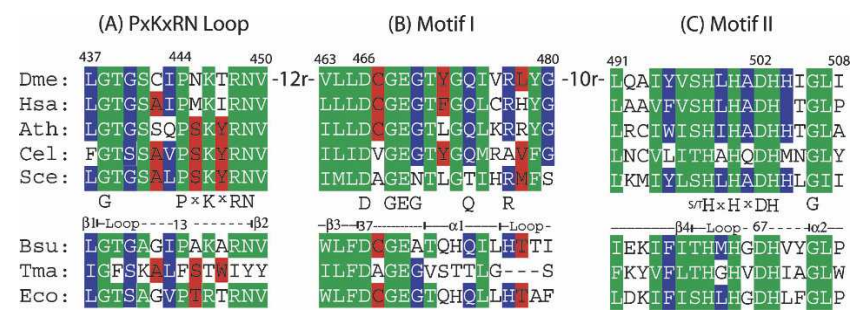

FIGURE 1. Alignment of selected homology blocks in tRNase $\mathrm{Z}^{\mathrm{L}}$ and tRNase $Z^{S}$ proteins. $(A)$ The PxKxRN loop, $(B)$ the region including Motif I, and $(C)$ the region surrounding Motif II. The top five aligned sequences are tRNase $\mathrm{Z}^{\mathrm{L}}$ s from Drosophila melanogaster, Homo sapiens, Arabidopsis thaliana, Caenorhabditis elegans, and Saccharomyces cerevisiae (accession nos. Q8MKW7, NP_060597.3, AAM51378, O4476, and NP013005.1, respectively). The bottom three sequences are tRNase $Z^{\mathrm{S}}$ s from B. subtilis, T. maritime, and E. coli (accession nos. P54548, NP_228673, and ZP_00726790, respectively). Designations below the tRNase $\mathrm{Z}^{\mathrm{L}}$ panels indicate the consensus, and coloring of residues indicates the extent of homology (green, identical; red, conserved in most cases; purple, similar). Numbering of selected residues above tRNase $\mathrm{Z}^{\mathrm{L}}$ panels is for fruit fly tRNase $\mathrm{Z}^{\mathrm{L}}$ based on the presumed translation initiation at an internal methionine (r24) as previously described in Zareen et al. 2005. Spacing between the three homology blocks presented is also conserved in the five tRNase $\mathrm{Z}^{\mathrm{L}}$ s. Residue numbers and designations above the bottom panel (indicating the positions of the secondary structure elements within the homology blocks) are based on Bsu tRNase $Z^{\mathrm{S}}$ (de la Sierra Gallay et al. 2005) 
Ishii et al. 2005; Kostelecky et al. 2006) show the same fold, two opposing clusters of the secondary structure elements $\beta \beta \beta \beta \alpha \beta \alpha \beta \alpha \beta$ in each subunit, in which the first $\beta$-strand is contributed by $\beta 16$. Composite views adapted from the model of $B$. subtilis tRNase Z (Fig. 2A-C, displayed using VMD from PDB ID 1Y44; de la Sierra-Gallay et al. 2005) represent the contribution that could be made by the PxKxRN loop and Motif I (potential regulatory domains) and Motif II to tRNase $\mathrm{Z}$ catalysis.

The alignments (Fig. 1) suggest that fundamental structural aspects ( $\beta$-sheet, $\alpha$-helix, loop size, and geometry) of the three blocks presented are conserved between their locations in the carboxy half of tRNase $Z^{\mathrm{L}}$ and the catalytic (A) subunit of B. subtilis tRNase $Z^{\mathrm{S}}$. The lack of structural information from the amino half of tRNase $Z^{\mathrm{L}}$ prevents use of the dimer interface of tRNase $Z^{S}$ to develop a model for catalysis by tRNase $Z^{\mathrm{L}}$, however, limiting detailed application of the tRNase $Z^{S}$ model to one active site (Motif II-V) in collaboration with one PxKxRN loop and one Motif I region.

From a variety of viewing angles the PxKxRN ( $\beta 1-\beta 2)$ loop appears to partially cover a cavernous opening to the active site (Motifs II-V) like a flap (Fig. 2B; de la SierraGallay et al. 2005). Characteristics attributed to this loop include rigidity conferred by $\mathrm{P} 13$, a conserved surface lysine (K15), and the ability to discriminate against C74 of mature tRNA as the first nucleotide of a CCA anti-determinant (de la Sierra-Gallay et al. 2005). Motif I is a single aspartate
A

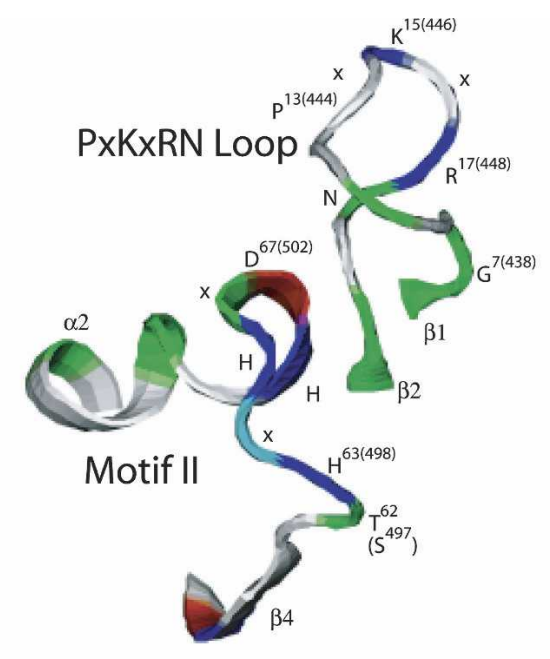

B

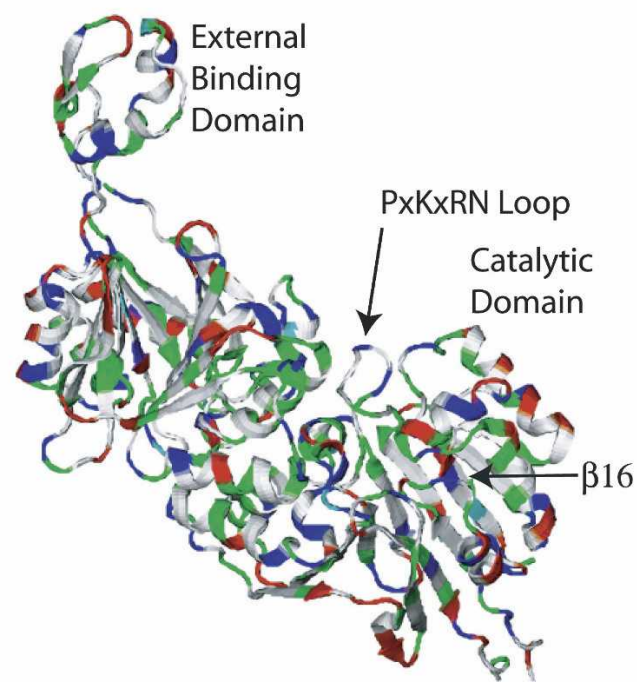

C

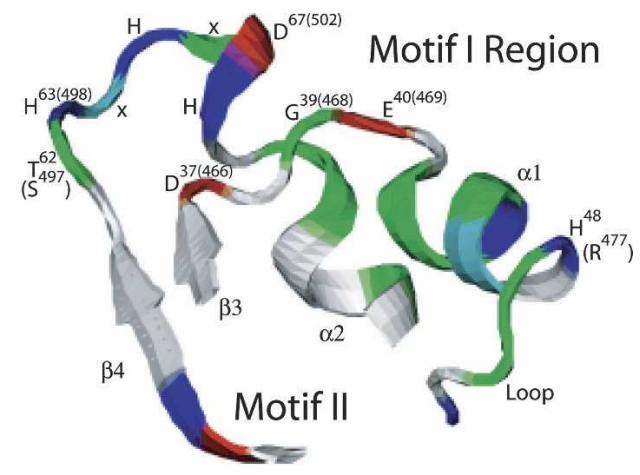

FIGURE 2. Views of tRNase $\mathrm{Z}$ taken from the B. subtilis structure (de la Sierra-Gallay et al. 2005; PDB ID 1Y44). Motif II and the other active site residues (Motifs III-V) are buried in the catalytic domain of the A subunit, and the PxKxRN loop appears to cover the entrance to the active site. (A) PxKxRN loop and Motif II. (B) entire Bsu. tRNase Z structure model in the same front view as in $A$ with the external binding domain in the upper left. (C) Motif I region and Motif II; the best view was obtained by rotating $\sim 180^{\circ}$ so that the external binding domain is in the upper right (a back view). The amino acid, $\alpha$-helix, and $\beta$-sheet nomenclature refer to the $B$. subtilis structure. Residue numbers in parentheses refer to the fruit fly protein. 
(D466 in D. melanogaster tRNase $\mathrm{Z}^{\mathrm{L}}$ ) at the start of the $\beta 3-$ $\beta 1$ loop (D37 in B. subtilis tRNase Z [de la Sierra-Gallay et al. 2005]; and D25 in T. maritima tRNase $Z$ [Ishii et al. 2005]), which is conserved in both ${ }^{\mathrm{L}}$ and ${ }^{\mathrm{S}}$ tRNase Zs (Fig. $1 B)$. D37, found at the periphery of important residues surrounding the inner circle of Motifs II-V, which directly bind the two divalent metals (de la Sierra-Gallay et al. 2005), was essential for tRNase $Z$ activity (Minagawa et al. 2004). The region including Motif I consists of $\beta$-strand, loop, $\alpha$-helix, and loop (Figs. 1B, 2C). Two residues in $\alpha 1$ were suggested to determine the unusual cleavage site of T. maritima tRNase $\mathrm{Z}$ (after transcriptionally embedded CCA) (Minagawa et al. 2004), corresponding in position to D. melanogaster Y472 and Q474 (based on alignments in Fig. 1B). We therefore performed a detailed mutational analysis of the PxKxRN loop and Motif I region.

\section{Expressed affinity purified soluble tRNase $\mathbf{Z}$}

In both Figure 1 and Figure 2, $\mathrm{A}$ and $\mathrm{C}$, the residues displayed are those that were subjected to Ala-scanning analysis (14 in the PxKxRN loop and 18 in the Motif I region). Wild-type and variant fruit fly tRNase Z's were constructed, baculovirus-expressed, and affinity-purified (Fig. 3; Zareen et al. 2005; see Materials and Methods). Yield and purity of soluble tRNase $\mathrm{Z}$ were sufficient to perform the efficiency and kinetic experiments. In Figure 3, $\sim 0.5 \mu \mathrm{g}$ of each protein was loaded from the first step of a dilution series. The values for enzyme concentration used

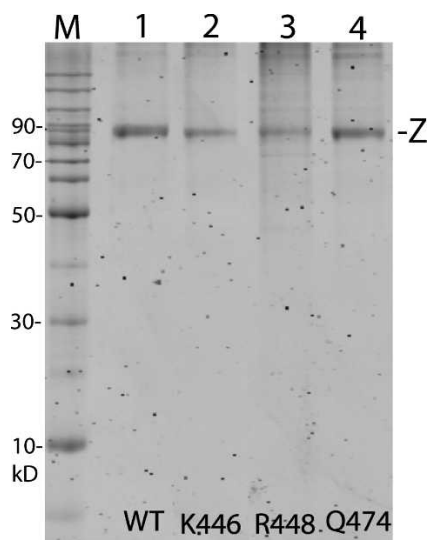

FIGURE 3. Baculovirus expression of fruit fly tRNase Z. Wild-type and mutant fruit fly tRNase Zs (indicated with $-\mathrm{Z}$ to the right of gel panel) were affinity-purified, electrophoresed on a $10 \%$ polyacrylamide SDS gel, stained with Sypro orange (Molecular Probes), and scanned for fluorescence with a Typhoon imager. The variant tRNase Z's presented (K446A, R448A, and Q474A, as designated below panel) were selected based on their effects on processing (see Fig. 3). Approximately $0.5 \mu \mathrm{g}$ of each tRNase $\mathrm{Z}$ sample was loaded. The enzyme concentrations used in kinetic experiments were determined from the protein gel lanes with ImageQuant to obtain $\mathrm{k}_{\mathrm{cat}}$ from $\mathrm{V}_{\max }$. Size designations are given for the marker lane to the left of the panel. Fruit fly tRNase $\mathrm{Z}$ displays an apparent molecular weight of $\sim 90 \mathrm{kDa}$, slightly larger than its predicted molecular weight of $83 \mathrm{kDa}$. to calculate processing efficiency and $\mathrm{k}_{\mathrm{cat}}$ were refined with protein concentrations determined by scanning these protein gel lanes (Zareen et al. 2005; see Materials and Methods). The substitutions illustrated and several others in the PxKxRN loop and Motif I have substantial effects on processing efficiency and kinetics (see below).

\section{Kinetics of pre-tRNA ${ }^{\mathrm{His}}$ processing}

Methods and data quality of processing kinetic analysis are illustrated in Figure 4. Fruit fly pre-tRNA ${ }^{\text {His }}$, the tRNase $\mathrm{Z}$ substrate used throughout (Frendewey et al. 1985; Levinger et al. 1995), is a 108-nt transcript consisting of a 72-nt tRNA (to the discriminator) and a 36-nt $3^{\prime}$-end trailer (substrate and tRNA product bands are marked on the right side of Fig. 4B). Processing efficiencies of enzymes with single residue substitutions were evaluated using a range of enzyme concentrations with labeled substrate and no added unlabeled substrate (data not shown; see Materials and Methods; Zareen et al. 2005). Concentrations of tRNase Zs with impairing substitutions were thereby adjusted to approximately match the overall extent of processing in the kinetic analysis. For example, tRNase $\mathrm{Z}$ with the R448A substitution was used at a concentration of $4.5 \mathrm{nM}, \sim 300$ times more concentrated than the $12.5 \mathrm{pM}$ wild-type tRNase Z (Fig. 4, cf. B and A). Increasing variant enzyme concentration could have unknown effects in addition to accelerating the assay, but the reactions catalyzed by enzyme variants with impairing substitutions all clearly fit the Michaelian model (Fig. 4C; data not shown).

Eadie-Hofstee plots for four variants are superimposed in Figure 4C. Interestingly, two of the variants presented here (K446A in the PxKxRN loop and Q474A in Motif I) display a $\mathrm{k}_{\mathrm{M}}$ significantly higher than that of the wild-type enzyme (slope in Fig. 4C), which was not observed with any of the substitutions in Motif II (Zareen et al. 2005). $V_{\max }$ (the intercepts on the ordinate in Fig. 4C) and the concentration of enzyme used in each reaction (shown in Fig. 4A-C and in the fourth column of D) were used to calculate $\mathrm{k}_{\text {cat }}$ (rightmost column in Fig. 4D). In these representative examples of variant processing kinetics, in addition to the effects on $\mathrm{k}_{\mathrm{M}}$, variant $\mathrm{k}_{\mathrm{cat}} \mathrm{s}$ were all reduced (three-, $\sim 60$-, and 800-fold in the case of K446A, Q474A, and R448A, respectively). These results (and additional experiments performed at least twice for each variant enzyme; data not shown) were used to determine mean $\mathrm{k}_{\text {cat }}, \mathrm{k}_{\mathrm{M}}$, and catalytic efficiency $\left(\mathrm{k}_{\mathrm{cat}} / \mathrm{k}_{\mathrm{M}}\right)$ including the standard errors for all measurements, and the effect on catalytic efficiency relative to wild type (Tables 1, 2, columns 2-5).

Results are presented graphically in Figures 5 and 6. In the bar graphs, $\mathrm{k}_{\mathrm{M}}$ relative to wild type (ordinate, linear scale) increased by up to eightfold for substitutions in the PxKxRN loop (K446A) (abscissa in Fig. 5A; cf. Fig. 4C,D 


\section{(A) 12.5 pM WT tRNase Z}

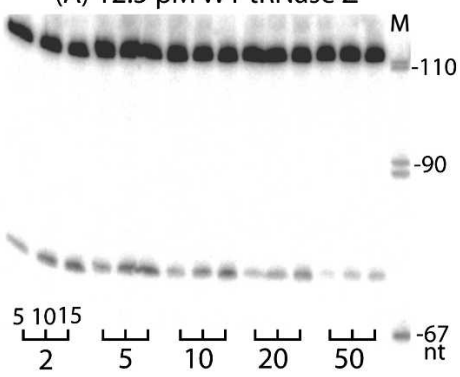

(B) 4.5 nM R448A tRNase Z

0
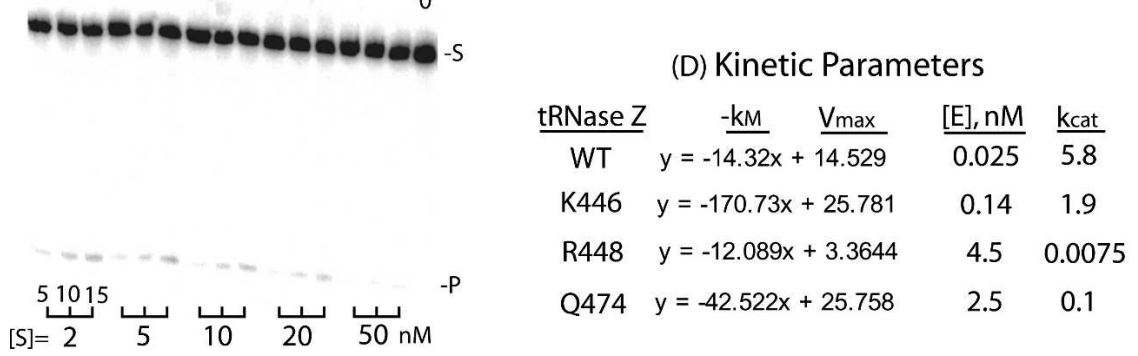

FIGURE 4. Kinetics of processing with wild-type and variant tRNase Z. Mutations analyzed are the same as in Figure 2. $(A, B)$ The processing in a kinetic experiment with wild-type and R448A tRNase Z. Reactions were sampled after 5,10 , and $15 \mathrm{~min}$ as designated by the brackets below the data panels. The concentrations of tRNase Z (12.5 pM for wild type and $4.5 \mathrm{nM}$ for $\mathrm{R} 448 \mathrm{~A}$, designated above the panels) were chosen to produce a similar extent of processing (\% product/minute of reaction, equivalent to $\mathrm{V} /[\mathrm{S}]$ ). The substrate concentrations, adjusted by adding unlabeled fruit fly pre-tRNA ${ }^{\text {His }}$, were $2,5,10,20$, and $50 \mathrm{nM}$, as indicated below the panels. (C) Eadie-Hofstee plots for wild type, K446, R448, and Q474. Concentrations of enzymes used are indicated on the plot. $(D)$ Kinetic parameters from the data presented in $A-C$. Equations presented for the enzymes (columns labeled $-\mathrm{k}_{\mathrm{M}}, \mathrm{V}_{\max }$, with units of $\times 10^{-9}$ $\mathrm{M}$ and $\times 10^{-11} \mathrm{M} / \mathrm{min}$., respectively) are taken from the Excel plots $(C)$. $\mathrm{k}_{\mathrm{cat}}$ is calculated from $\mathrm{V}_{\max }$ using the value for [E] determined from the protein gel lane for each mutant (Fig. 1) based on the dilution factor used in the experiment.

and Table 1) and by up to 15 -fold for substitutions in Motif I (R477A) (see Table 2; Fig. 6A). The relative effects of the substitutions on $\mathrm{k}_{\mathrm{cat}}$ and $\mathrm{k}_{\mathrm{cat}} / \mathrm{k}_{\mathrm{M}}$ are presented using an inverse log scale (Figs. 5, 6, ordinate in panels B,C) to display the changes that reduce processing efficiency (relative increase in $\mathrm{k}_{\mathrm{M}}$ and decrease in $\mathrm{k}_{\mathrm{cat}}$ ) in the same (positive) direction and because the effect of a single substitution on $\mathrm{k}_{\mathrm{cat}}$ can be up to 1000 -fold greater than its effect on $\mathrm{k}_{\mathrm{M}}$ (e.g., G438A and R448A in the PxKxRN loop) (Fig. 5). In the most extreme cases (G438A in the PxKxRN loop [Table 1; Fig. 5C]; D466A and R477A in the Motif I region [Table 2; Fig. 6C]) processing efficiency was reduced between 1000- and 10,000-fold, comparable to the greatest reductions found with substitutions in Motif II (Zareen et al. 2005). Several mutants have been observed in which Ala substitution impairs reaction efficiency between 1000- and 10,000-fold, including D466, H500, and D502 (Zareen et al. 2005; this report), which were found by others to be essential for activity (Minagawa et al. 2004; Spath et al. 2005). These discrepancies could arise from differences in expression systems, assay methods, reaction optimization, and the form of tRNase $\mathrm{Z}\left({ }^{\mathrm{L}}\right.$ or $\left.{ }^{\mathrm{S}}\right)$. Expression and assay methods reported here allow the kinetic parameters to be obtained from mutant tRNAse Zs impaired by up to $10^{5}$-fold relative to wild type. Some of the substitutions have slight effects, but both $\mathrm{k}_{\mathrm{cat}}$ and $\mathrm{k}_{\mathrm{cat}} / \mathrm{k}_{\mathrm{M}}$ were reduced relative to wild type in all cases (the values on the ordinate in panels $\mathrm{B}, \mathrm{C}$ of Figs. 5, 6 are all positive).

Unlike the Motif II substitutions that reduce catalytic efficiency without affecting $k_{\mathrm{M}}$, substitutions in the PxKxRN loop and Motif I region can affect $\mathrm{k}_{\text {cat }}$, $\mathrm{k}_{\mathrm{M}}$ or both, as indicated by the symbols below the abscissas in Figures 5 and 6 $\left(\square, \boldsymbol{\square}\right.$ : moderate or great increase in $\mathrm{k}_{\mathrm{M}}$, respectively; $\bigcirc, \bigcirc$ : moderate or great decrease in $k_{\text {cat }}$ ), as follows: G438A and C442A cause a moderate (threeto fourfold) increase in $\mathrm{k}_{\mathrm{M}}$ and $\mathrm{K} 446 \mathrm{~A}$ causes a larger (about eightfold) increase in $\mathrm{k}_{\mathrm{M}}$ (PxKxRN loop) (Table 1; Fig. 5A); D466A and R477A (Motif I region) (Table 2; Fig. 6A) also cause large increases in $k_{M}$ (7.5- and 15-fold, respectively). The moderate and large decreases in $\mathrm{k}_{\text {cat }}$ in the PxKxRN loop arise from the substitutions G440A, N445A, and N449A (between 100- and 1000-fold) and G438A and R448A $(>1000 \times)$, respectively (Table 1; Fig. $5 \mathrm{~B})$, and those in the Motif I region arise from C467A, G470A, Q474A, Y479A, and G480A (100- to 1000-fold) and D466A, G468A, E469A, and R477A (>1000-fold) (Table 2 and Fig. 6B, respectively).

In the carboxy half of the PxKxRN loop, the order of importance of the conserved residues is $\mathrm{P}^{444}<\mathrm{K}^{446}<\mathrm{R}^{448}$ $>\mathrm{N}^{449}$ (Fig. 5). The effect of the R448A substitution on catalytic efficiency is 10 times greater than that of K446A; K446A acts principally through an increase in $\mathrm{k}_{\mathrm{M}}$ while $\mathrm{R} 448 \mathrm{~A}$ acts only by causing a decrease in $\mathrm{k}_{\text {cat }}$. Interestingly, substitution of R448 causes a reproducible decrease in $\mathrm{k}_{\mathrm{M}}$ in addition to the large decrease in $\mathrm{k}_{\text {cat }}$. Substitution of N445 causes an even larger decrease in $\mathrm{k}_{\mathrm{M}}$; these are the only two residues that do so.

\section{CCA at the $3^{\prime}$ end of mature tRNA is a tRNase $Z$ anti-determinant that reduces $\mathrm{k}_{\text {cat }} \mathbf{8 0}$-fold}

The efficiencies with which mature tRNA with CCA at the $3^{\prime}$ end and pre-tRNA with the short sequence-AUG (taken from sequence of the natural pre-tRNA ${ }^{\text {His }} 3^{\prime}$-end trailer) can be processed by tRNase $\mathrm{Z}$ were compared. The $\sim 1$-nt greater length of the -AUG substrate (Fig. 7; see Materials 
TABLE 1. PXKXRN loop variant processing kinetics

\begin{tabular}{|c|c|c|c|c|}
\hline 1 & 2 & 3 & 4 & 5 \\
\hline Enzyme & $\mathrm{K}_{\text {cat }}{ }^{\mathrm{a}}$ & $\mathrm{K}_{M}{ }^{\mathrm{b}}$ & $\mathrm{K}_{\mathrm{cat}} / \mathrm{K}_{\mathrm{M}}{ }^{\mathrm{c}}$ & $\operatorname{Re} K_{\text {cat }} / K_{M}{ }^{d}$ \\
\hline WT & $4.99 \pm 0.57$ & $1.98 \pm 0.16$ & $2.52 \pm 0.18$ & 1 \\
\hline L437 & $1.15 \pm 0.23$ & $3.33 \pm 0.18$ & $0.35 \pm 0.09$ & 0.14 \\
\hline G438 & $0.0034 \pm 0.0012$ & $6.92 \pm 2.75$ & $0.00061 \pm 0.0002$ & 0.00021 \\
\hline T439 & $1.8 \pm 0.6$ & $3.84 \pm 1.34$ & $0.47 \pm 0.01$ & 0.38 \\
\hline G440 & $0.19 \pm 0.1$ & $2.69 \pm 0.79$ & $0.067 \pm 0.017$ & 0.027 \\
\hline S441 & $0.89 \pm 0.11$ & $3.95 \pm 0.32$ & $0.22 \pm 0.009$ & 0.11 \\
\hline C442 & $0.75 \pm 0.12$ & $7.30 \pm 2.70$ & $0.113 \pm 0.027$ & 0.083 \\
\hline 1443 & $0.71 \pm 0.34$ & $2.14 \pm 0.18$ & $0.32 \pm 0.13$ & 0.12 \\
\hline P444 & $1.42 \pm 0.87$ & $2.17 \pm 0.97$ & $0.61 \pm 0.12$ & 0.19 \\
\hline N445 & $0.25 \pm 0.08$ & $0.77 \pm 0.08$ & $0.34 \pm 0.14$ & 0.21 \\
\hline K446 & $2.01 \pm 0.20$ & $15.8 \pm 6.2$ & $0.19 \pm 0.09$ & 0.04 \\
\hline T447 & $1.60 \pm 0.40$ & $1.95 \pm 0.05$ & $0.82 \pm 0.19$ & 0.54 \\
\hline R448 & $0.0049 \pm 0.0026$ & $1.04 \pm 0.17$ & $0.0045 \pm 0.0019$ & 0.0016 \\
\hline N449 & $0.16 \pm 0.12$ & $3.75 \pm 0.75$ & $0.038 \pm 0.024$ & 0.012 \\
\hline V450 & $1.09 \pm 0.28$ & $2.87 \pm 0.81$ & $0.39 \pm 0.05$ & 0.25 \\
\hline
\end{tabular}

Averages are presented of two or more Michaelis-Menten experiments with tRNase Z processing of pre-tRNA ${ }^{\text {His }}$ using each of the PxKxRN mutants (column 1). Columns 1-5 are identified in the second row.

${ }^{a} \min ^{-1}$

${ }^{\mathrm{b}} \times 10^{-8} \mathrm{M}$.

${ }^{\mathrm{c}} \times 10^{8} \mathrm{M} / \mathrm{min}$.

${ }^{d} \operatorname{Re} K_{\text {cat }} / K_{M}$ ( $n$-fold reduction) refers to the relative ratio of processing efficiencies: $K_{\text {cat }} / K_{M}$ (variant) $/ K_{\text {cat }} / K_{M}$ (wild type) in parallel kinetic experiments.

and Methods) should not affect the results or interpretation of the -CCA anti-determinant experiments; the first three nucleotides of the $3^{\prime}$-end trailer following the discriminator have the most pronounced effect on $t R N a s e ~ Z$ reaction, and the greatest anti-determinant effect was observed with the first two (-CC) (Mohan et al. 1999). Approximately 80 times more enzyme is required with mature tRNA (-CCA) (Fig. $7 \mathrm{~B}$ ) to achieve a processing time course comparable to that observed with natural sequence (Fig. 7A).

The results of the relative efficiency experiments (Fig. 7) were confirmed and extended by performing kinetics with the pre-tRNA ( $3^{\prime}$-AUG) and mature tRNA ( $3^{\prime}$-CCA) substrates (Table 3 ). The $\mathrm{k}_{\text {cat }}$ for $3^{\prime}$-AUG substrate is $\sim 4.5$ times higher than that for $\mathrm{tRNA}^{\mathrm{His}}$ with a $36-\mathrm{nt}$ $3^{\prime}$-end trailer, and the $\mathrm{k}_{\mathrm{M}}$ is around two times higher for a combined slightly greater than twofold increase in $\mathrm{k}_{\mathrm{cat}} / \mathrm{k}_{\mathrm{M}}$ (cf. Table 3, line 1, and Table 1, line 1). $\mathrm{k}_{\text {cat }}$ for the -CCA substrate is reduced 80 -fold relative to the -AUG substrate (the pertinent comparison) without an effect on $\mathrm{k}_{\mathrm{M}}$. The CCA anti-determinant effect, an 80 -fold reduction in $\mathrm{k}_{\text {cat }}$, thus arises from a component of fruit fly tRNase $\mathrm{Z}$ and does not require an ancillary factor.

\section{DISCUSSION}

\section{Five regions of pre-tRNA involved in recognition and catalysis by $t R$ Nase $Z$}

The pre-tRNA can be divided into five regions that contribute to tRNase $\mathrm{Z}$ binding and catalysis: the $\mathrm{T}$ arm, the acceptor stem, the scissile bond, the three nucleotides following the discriminator, and the rest of the $3^{\prime}$-end trailer (designated A-E, respectively, in Fig. 8). According to this model, the PxKxRN loop and Motif I, which are principally involved in CCA anti-determination and acceptor stem binding (through regions D and B in the substrate, respectively), are located close to each other and to the active site, and thus close to the scissile bond of the substrate (C). Structural and mutational data suggest that the T arm of the tRNA substrate (A) binds to the external binding domain of the enzyme while the $3^{\prime}$-end trailer (E) extends beyond the active site in an RNA exit channel. The significant contribution of Motif I to binding the acceptor stem, as well as the distribution of surface charge along the enzyme, suggest that much of the tRNA substrate specificity resides near the active site, with the external binding domain and exit channel providing additional stability.

Substitutions in Motif II that reduce $\mathrm{k}_{\text {cat }}$ by as much as 7500-fold have no effect on $\mathrm{k}_{\mathrm{M}}$ (Zareen et al. 2005); this region is thus concerned only with catalysis and the substrate recognition and binding components must reside elsewhere. The tRNase Z structure (de la Sierra-Gallay et al. 2005; Ishii et al. 2005; Kostelecky et al. 2006) and analysis of multiple sequence alignments and deletions (Schilling et al. 2005) suggest that an external binding domain remote from the catalytic core of tRNase $\mathrm{Z}$ is concerned with pre-tRNA recognition and binding. The minimal substrate for enzymes involved with pre-tRNA metabolism (RNase P, tRNase Z, CCA-adding enzyme) is a minihelix consisting of the coaxially stacked acceptor stem and $\mathrm{T}$ arm (McClain et al. 1987; 
TABLE 2. Motif I variant processing kinetics

\begin{tabular}{|c|c|c|c|c|}
\hline 1 & 2 & 3 & 4 & 5 \\
\hline Enzyme & $\mathrm{K}_{\text {cat }}{ }^{\mathrm{a}}$ & $K_{M}{ }^{b}$ & $\mathrm{~K}_{\mathrm{cat}} / \mathrm{K}_{\mathrm{M}}{ }^{\mathrm{c}}$ & $\operatorname{Re} K_{\text {cat }} / K_{M}{ }^{c}$ \\
\hline WT & $4.99 \pm 0.58$ & $1.98 \pm 0.16$ & $2.52 \pm 0.18$ & 1 \\
\hline V463 & $0.48 \pm 0.05$ & $1.95 \pm 0.39$ & $0.26 \pm 0.08$ & 0.17 \\
\hline L464 & $0.86 \pm 0.37$ & $2.16 \pm 0.14$ & $0.38 \pm 0.16$ & 0.20 \\
\hline L465 & $0.50 \pm 0.29$ & $2.30 \pm 0.35$ & $0.24 \pm 0.16$ & 0.10 \\
\hline D466 & $0.010 \pm 0.002$ & $14.8 \pm 4.9$ & $0.0017 \pm 0.0009$ & 0.00066 \\
\hline C467 & $0.36 \pm 0.03$ & $1.69 \pm 0.29$ & $0.22 \pm 0.02$ & 0.12 \\
\hline G468 & $0.007 \pm 0.002$ & $3.70 \pm 0.38$ & $0.0019 \pm 0.0004$ & 0.0011 \\
\hline E469 & $0.012 \pm 0.0005$ & $1.52 \pm 0.04$ & $0.0079 \pm 0.0005$ & 0.0034 \\
\hline G470 & $0.11 \pm 0.008$ & $1.07 \pm 0.26$ & $0.11 \pm 0.025$ & 0.075 \\
\hline T471 & $0.68 \pm 0.16$ & $2.21 \pm 0.40$ & $0.32 \pm 0.06$ & 0.21 \\
\hline Y472 & $2.39 \pm 1.03$ & $6.24 \pm 2.39$ & $0.45 \pm 0.14$ & 0.24 \\
\hline G473 & $0.74 \pm 0.24$ & $2.10 \pm 0.80$ & $0.36 \pm 0.02$ & 0.13 \\
\hline Q474 & $0.12 \pm 0.045$ & $4.95 \pm 0.83$ & $0.022 \pm 0.0053$ & 0.0013 \\
\hline 1475 & $1.65 \pm 0.65$ & $2.55 \pm 0.45$ & $0.63 \pm 0.15$ & 0.19 \\
\hline V476 & $1.27 \pm 0.43$ & $2.44 \pm 0.64$ & $0.51 \pm 0.04$ & 0.16 \\
\hline R477 & $0.032 \pm 0.010$ & $29.3 \pm 2.11$ & $0.0011 \pm 0.0003$ & 0.00038 \\
\hline L478 & $1.12 \pm 0.48$ & $3.23 \pm 1.58$ & $0.36 \pm 0.03$ & 0.09 \\
\hline Y479 & $0.37 \pm 0.02$ & $1.62 \pm 0.24$ & $0.24 \pm 0.05$ & 0.14 \\
\hline G480 & $0.35 \pm 0.14$ & $1.59 \pm 0.20$ & $0.21 \pm 0.06$ & 0.12 \\
\hline
\end{tabular}

Averages are presented of two or more Michaelis-Menten experiments with tRNase Z processing of pre-tRNA ${ }^{\text {His }}$ using each of the mutants including and extending beyond Motif I (column 1). Columns 1-5 are identified in the second row.

${ }^{\mathrm{a}} \min ^{-1}$.

${ }^{\mathrm{b}} \times 10^{-8} \mathrm{M}$.

${ }^{\mathrm{c}} \times 10^{8} \mathrm{M} / \mathrm{min}$.

${ }^{d} \operatorname{Re} K_{\text {cat }} / K_{M}$ ( $n$-fold reduction) refers to the relative ratio of processing efficiencies: $K_{\text {cat }} / K_{M}$ (variant) $/ K_{\text {cat }} / K_{M}$ (wild type) in parallel kinetic experiments.

Levinger et al. 1998; Shi et al. 1998). A specific interaction can therefore be proposed between the external binding domain of tRNase Z (Schilling et al. 2005) and the T arm of tRNA (A in Fig. 8), which are distal to the active site of the enzyme and the scissile bond of the substrate, respectively.

Significant increases in $\mathrm{k}_{\mathrm{M}}$ arise from substitutions in the PxKxRN loop (G438, C442, K446) (Fig. 5A) and Motif I region (D466 and especially R477) (Fig. 6A), which are too close to the active site to interact with the $\mathrm{T}$ arm of the substrate (Fig. 2). Effects of acceptor stem substitutions in the pre-tRNA substrate on tRNase $\mathrm{Z}$ reaction efficiency and kinetics have been previously reported (Marchfelder et al. 1996; Mohan and Levinger 2000; Levinger et al. 2004a; Yan et al. 2006), suggesting that a component of the enzyme could clamp the acceptor stem (B in Fig. 8).

Involvement of tRNase $\mathrm{Z}$ with the $3^{\prime}$-end trailer could have two components: a CCA anti-determinant domain, which distinguishes between mature tRNA with -CCA at the $3^{\prime}$ end and pre-tRNAs with $3^{\prime}$-end trailers possessing a sequence other than CCA following the discriminator (Fig. 8D), and a clamp for the $3^{\prime}$-end trailer (E in Fig. 8) which then productively binds proper substrates, stabilizing the scissile bond in the active site. A narrow exit channel was posited in interpretation of the B. subtilis model (de la Sierra-Gallay et al. 2005), suggesting a binding domain for the $3^{\prime}$-end trailer. Early support for such an exit channel was provided by analysis of pre-tRNAs with transcription- ally embedded CCA (Nashimoto 1997), in which increasing the length of the $3^{\prime}$-end trailer partially rescues mammalian tRNase $\mathrm{Z}$ catalysis.

\section{Function of the PxKxRN loop is dominated by glycine at the amino boundary (G438) and the internal arginine (R448)}

The great effect of the G438 substitution (a modest increase in $\mathrm{k}_{\mathrm{M}}$ and a much larger reduction in $\mathrm{k}_{\text {cat }}$ combine to reduce $\mathrm{k}_{\mathrm{cat}} / \mathrm{k}_{\mathrm{M}}$ 5000-fold) (Table 1; Fig. 5) suggests that the function of the entire loop can be ruined by replacement of this residue at the $\beta 1$-loop boundary by allowing the secondary structure to extend beyond its natural end, drastically affecting shape and function of the PxKxRN loop. Replacement of P444 in mid-loop has comparatively little effect; P444 thus apparently does not contribute substantially to function of the PxKxRN loop, although it is conserved (Fig. 1A) and introduces a rigid bend (Fig. 2; de la Sierra-Gallay et al. 2005).

While it would be premature to offer a detailed model for regulation of tRNase $\mathrm{Z}$ activity, the conserved basic residues K446 and R448 may conduct surveillance on C74 and C75 as part of a CCA anti-determinant, interacting with region $\mathrm{D}$ in Figure 8. Analysis of processing kinetics using tRNA-C, -CC and -CCA showed that C75 (the second C) combined with C74 makes a greater contribution than C74 alone to the 


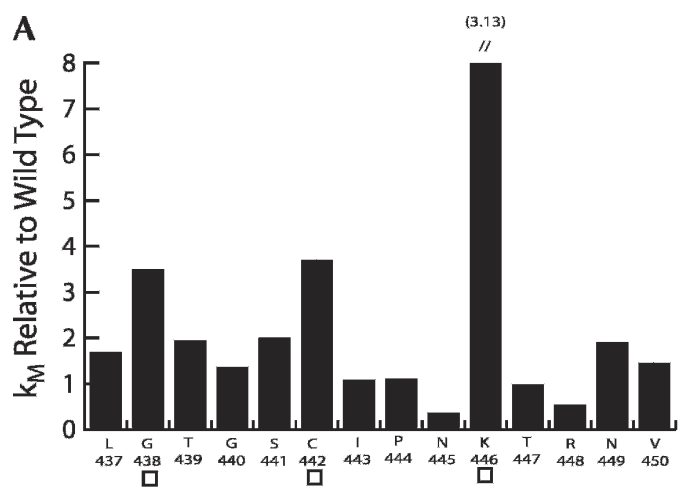

B

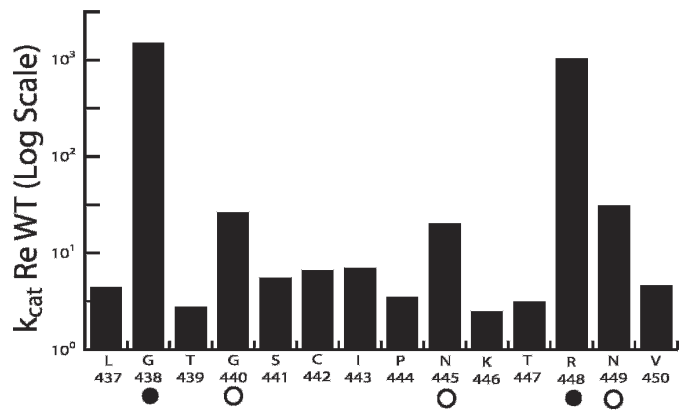

C

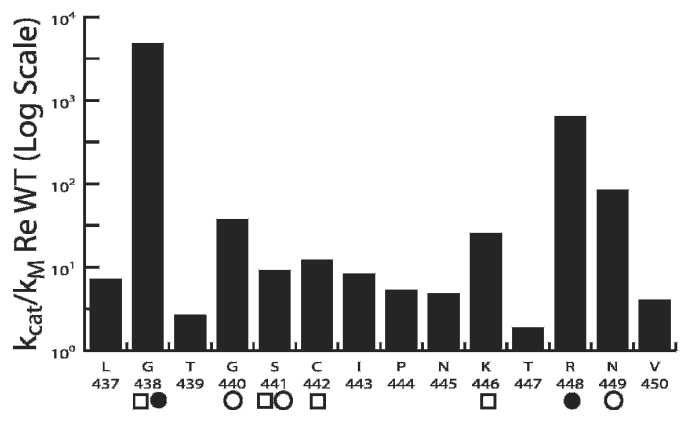

FIGURE 5. Graphical representation of the effects of substitutions in the PxKxRN loop on tRNase $\mathrm{Z}$ processing. $(A) \mathrm{k}_{\mathrm{M}}$ relative to wild type (from column 3 in Table 1) is presented on a linear scale. The wildtype residues substituted with alanine in the PxKxRN loop are identified on the abscissa. The line and the bar above each value on the bar graph indicate the standard error (from Table 1). (B) The inverse of $\mathrm{k}_{\mathrm{cat}}$ relative to wild type ( $\mathrm{n}$-fold decrease in $\mathrm{k}_{\mathrm{cat}}$, taken from column 2 in Table 1) is presented on a log scale. (C) The inverse of $\mathrm{k}_{\mathrm{cat}} / \mathrm{k}_{\mathrm{M}}$ relative to wild type (taken from column 5 in Table 1) is presented on a log scale. Conserved residues (taken from Fig. 1A) that affect one or more of the kinetic parameters are presented with symbols ( $\square, \bigcirc$ for the intermediate effects on $\mathrm{k}_{\mathrm{M}}$ and $\mathrm{k}_{\mathrm{cat}}$, respectively; X,@ for the greatest effects). Error bars are not presented in $B$ and $C$ but can be obtained from Table 1 .

CCA anti-determinant (Mohan et al. 1999), just as substitution of R448 more strongly reduces tRNase $\mathrm{Z}$ reaction efficiency than substitution of K446.

\section{The Motif I region consists of four structural elements}

Motif I consists of a single aspartate that is important for tRNase Z catalysis (D25 in T. martitima, D37 in B. subtilis, and D466 in D. melanogaster tRNase Z) (Minagawa et al.
2004; de la Sierra-Gallay et al. 2005; Ishii et al. 2005), but the region subjected to detailed mutagenesis analysis was extended in both directions to include $\beta 3$, the $\beta 3-\alpha 1$ loop, $\alpha 1$, and part of the $\alpha 1-\beta 4$ loop. Troughs are observed in the effects of substitutions on $\mathrm{k}_{\mathrm{cat}}$ and $\mathrm{k}_{\mathrm{cat}} / \mathrm{k}_{\mathrm{M}}$ coinciding with $\beta 3$ and $\alpha 1$ in the Motif I region except for Q474 (Fig. $6 \mathrm{~B}, \mathrm{C})$, suggesting that Ala substitutions at the boundaries and in connecting loops usually affect tRNase $\mathrm{Z}$ function more than those within these structural elements.

Replacement of Motif I D466 with Ala reduces catalytic efficiency $\left(\mathrm{k}_{\text {cat }} / \mathrm{k}_{\mathrm{M}}\right)$ 3750-fold due to a 7.5 -fold increase in $\mathrm{k}_{\mathrm{M}}$ and a 500 -fold reduction in $\mathrm{k}_{\text {cat }}$ relative to wild type. Substitutions in G468 and E469 close to the loop- $\alpha 1$ boundary also affect tRNase $\mathrm{Z}$ catalysis. Being strongly conserved (Fig. 1B), these residues might have both structural and electrostatic importance.
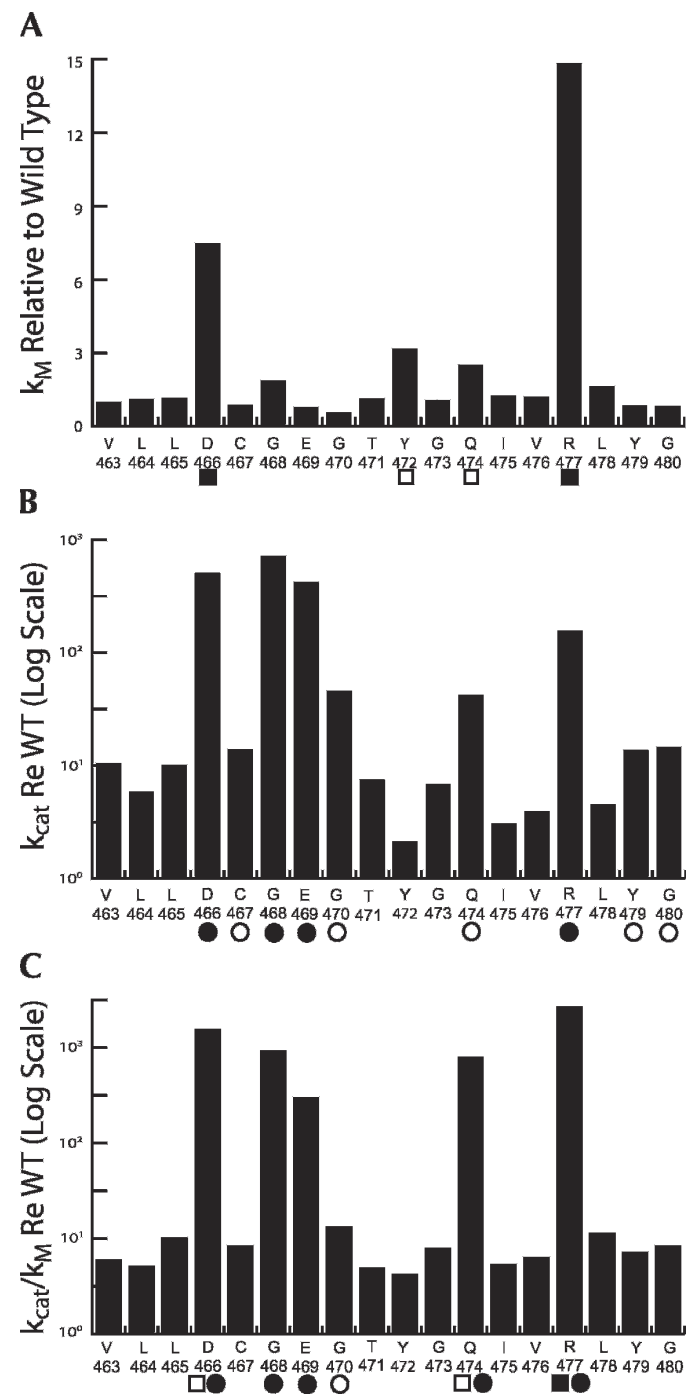

FIGURE 6. Graphical representation of the effects of substitutions in the Motif I region. The presentation of $\mathrm{k}_{\mathrm{M}}, \mathrm{k}_{\mathrm{cat}}$, and reaction efficiency $\left(\mathrm{k}_{\mathrm{cat}} / \mathrm{k}_{\mathrm{M}}\right)$ relative to wild type in $A-C$ and use of the symbols $\square, \bigcirc, \square, \bigcirc$ is the same as in Figure 5. 


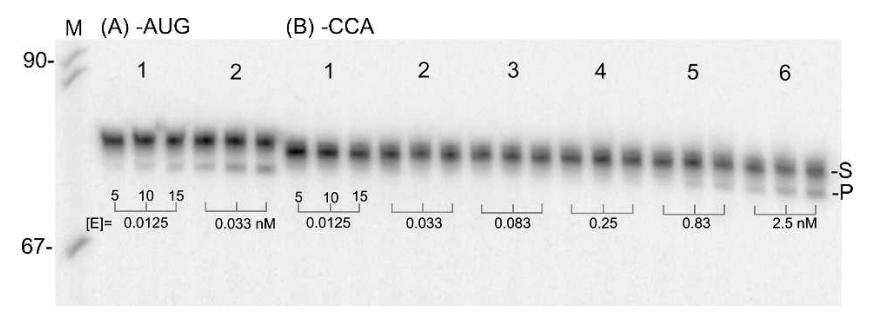

FIGURE 7. Effect of the CCA anti-determinant on efficiency of tRNase $\mathrm{Z}$ reaction. T7 runoff templates were designed to produce a pre-tRNA ${ }^{\text {His }}$ substrate with the natural sequence A-AUG or with A-CCA of mature tRNA following the discriminator base (A-). Unlabeled pre-tRNAs were transcribed, gel-purified, treated with shrimp alkaline phosphatase, labeled with $\gamma^{32} \mathrm{P}$-ATP using T4 polynucleotide kinase, and repurified (side designation $\mathrm{S}$ for substrate). These labeled pre-tRNAs were used in processing reactions sampled after 5, 10, and 15 min as indicated with vertical ticks below the product bands using enzyme concentrations of 0.0125 and $0.033 \mathrm{nM}$ in the reactions with the -AUG pre-tRNA and with $0.0125,0.033,0.083,0.25,0.83$, and $2.5 \mathrm{nM}$ in reactions with -CCA mature tRNA as indicated below the gel. Comparable processing of -AUG substrate with $0.033 \mathrm{nM}$ tRNase $\mathrm{Z}$ and of -CCA tRNA with $2.5 \mathrm{nM}$ tRNase $\mathrm{Z}$ suggests that the CCA anti-determinant reduces reaction efficiency $\sim 80$-fold.

Y472 and Q474 correspond in position with two residues in $\alpha 1$ that were suggested to be involved in determination of the unusual cleavage site of T. maritima tRNase Z (Minagawa et al. 2004). Substitution of $Y 472$ has practically no effect and Q474A has a moderate effect on both $\mathrm{k}_{\mathrm{M}}$ and $\mathrm{k}_{\mathrm{cat}}$, which combine to make it one of the most important residues in this region. Neither substitution affects the tRNase $\mathrm{Z}$ cleavage site in fruit fly tRNA ${ }^{\text {His }}$ (data not shown).

$\mathrm{R} 477$ is located at the opposite end of the Motif I region from D466 (the loop on the carboxy side of $\alpha 1$ ) (Fig. 2C). Spikes in $\mathrm{k}_{\mathrm{M}}$ arise from the D466 and R477 substitutions (Fig. $5 A$ ), while substitution of R477 has an even greater effect on efficiency than D466. While D466 has previously documented effects on catalysis (the equivalent conserved residue in $T$. maritima tRNase $\mathrm{Z}^{\mathrm{S}}$ ) (Minagawa et al. 2004), R477 must function differently, perhaps in binding the acceptor stem.

\section{Effects of CCA on tRNase $Z$ reaction}

The CCA anti-determinant causes $\mathrm{k}_{\text {cat }}$ to decrease 80 -fold without affecting $\mathrm{k}_{\mathrm{M}}$ (Table 3 ). In earlier experiments with
C-C-A added sequentially to the discriminator using unpurified fruit fly tRNase $\mathrm{Z}$ and tRNase $\mathrm{Z}$ purified from pig liver, decreases in $k_{\text {cat }}$ and increases in both $k_{M}$ and $k_{i}$ contributed to the CCA anti-determinant effect (Mohan et al. 1999). The discrepancy between present and earlier results could be explained if ancillary factors present in extracts but absent from expressed, affinity-purified tRNase $\mathrm{Z}$ can bind tRNA, affecting $\mathrm{k}_{\mathrm{M}}$ and $\mathrm{k}_{\mathrm{i}}$ for tRNase $\mathrm{Z}$.

Since part of the CCA anti-determinant function may reside in the PxKxRN loop, its absence from T. maritima tRNase Z (Fig. 1A) could explain how this enzyme interacts atypically with CCA-containing pre-tRNAs after CCA (Minagawa et al. 2004; de la Sierra-Gallay et al. 2005). Interestingly, the first tRNase $\mathrm{Z}$ expressed and characterized (trzl from A. thaliana, a tRNase $Z^{S}$ ) (Schiffer et al. 2002) also lacks a PxKxRN loop, which could be why it lacks a tRNase $Z$ antideterminant (Schiffer et al. 2003). A. thaliana tRNase $Z^{\mathrm{L}}$ s, on the other hand, have a PxKxRN loop (Fig. 1A). M. jannaschii tRNase $\mathrm{Z}$ has a PxKxRN loop so the lack of a CCA antideterminant effect with this enzyme (Schiffer et al. 2003) must have a different explanation. tRNase $\mathrm{Z}$ processing experiments performed elsewhere typically use a 1000 - to 100,000-fold higher enzyme concentration than that used here. The differential CCA effect (Fig. 7) can be reduced or even eliminated at a high enzyme concentration (data not shown), which could explain why no effect of -CCA was observed with $M$. jannaschii tRNase Z.

\section{Characteristics of tRNase $Z$ distinguish it from other members of the $\beta$-lactamase family that have been suggested to be endoribonucleases}

Important endoribonucleases that for many years eluded identification may be cousins of tRNase $\mathrm{Z}$ in the $\beta$-lactamase family, including CPSF73 (implicated in premRNA, including histone pre-mRNA, $3^{\prime}$ end processing) (Ryan et al. 2004; Dominski et al. 2005a) and the CPSF73like integrator protein (suggested to be involved in snRNA $3^{\prime}$ end maturation) (Baillat et al. 2005; Dominski et al. 2005b). These proteins only function as site-specific endonucleases in concert with large macromolecular assemblies consisting of many additional protein subunits, while

TABLE 3. tRNase $Z$ processing kinetics with pre-tRNA ${ }^{\text {His }}$-AUG and -CCA substrates

\begin{tabular}{|c|c|c|c|c|}
\hline 1 & 2 & 3 & 4 & 5 \\
\hline Substrate & $\mathrm{K}_{\mathrm{cat}}{ }^{\mathrm{a}}$ & $\mathrm{K}_{M}{ }^{\mathrm{b}}$ & $\mathrm{K}_{\text {cat }} / \mathrm{K}_{M}{ }^{\mathrm{c}}$ & $\operatorname{Re} \mathrm{K}_{\text {cat }} / \mathrm{K}_{\mathrm{M}}{ }^{\mathrm{d}}$ \\
\hline tRNA ${ }^{\text {His }}$-AUG & $21.9 \pm 3.65$ & $3.94 \pm 0.30$ & $5.52 \pm 5.15$ & 1 \\
\hline tRNA $^{\mathrm{His}}$-CCA & $0.293 \pm 0.107$ & $4.01 \pm 1.14$ & $0.072 \pm 0.065$ & 0.013 \\
\hline
\end{tabular}




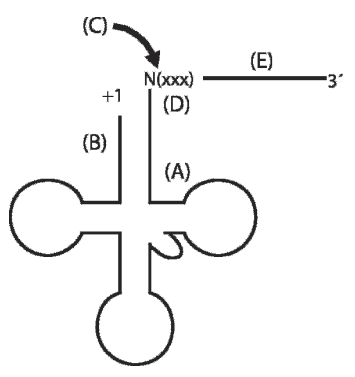

FIGURE 8. A pre-tRNA can be divided into five parts, each of them expected to contribute differently to tRNase $Z$ recognition and catalysis. (A) The $\mathrm{T}$ arm; (B) acceptor stem; (C) tRNase $\mathrm{Z}$ processing site; (D) three nucleotides following the cleavage site ( $\mathrm{xxx}=\mathrm{CCA}$ of mature tRNA or "not-CCA" of a pre-tRNA); (E) additional sequence of the $3^{\prime}$-end trailer.

tRNase $\mathrm{Z}$ is apparently a soloist. Distinguishing features of tRNase $Z$ include the external substrate recognition and binding domain (in the amino half of tRNase $Z^{\mathrm{L}}$ ), the PxKxRN loop, and Motifs I-V, which directly recognize the mutually complementary structural landmarks common to tRNAs (Fig. 8). Only weak homologies are observed between tRNase $\mathrm{Z}$ and its cousins outside of the His domain (Motif II); identification of the substrate recognition in the case of the other putative endoribonucleases thus requires more complex RNA-protein interactions.

\section{Future prospects}

Since the substitutions in the PxKxRN loop and Motif I region reveal residues that unexpectedly contribute to the catalytic efficiency of tRNase $Z$, it will be of interest to apply this scanning method to the homology blocks on the carboxy side of Motif II (e.g., Motifs III-V) and to the external recognition and binding domain in the amino half of tRNase $\mathrm{Z}^{\mathrm{L}}$. Additionally, the $\sim 10$-fold increases in $\mathrm{k}_{\mathrm{M}}$ arising from some of the single residue substitutions suggest direct contacts between enzyme and substrate that could be identified (as in Kühn-Hölsken et al. 2005). To finally understand the CCA anti-determinant it will be critical to solve the structures of $t R N A-t R N a s e ~ Z$ complexes.

\section{MATERIALS AND METHODS}

\section{Construction, expression, and affinity purification of tRNase $Z$ variants}

Procedures for site-specific mutagenesis, baculovirus expression, and affinity purification of fruit fly tRNase $\mathrm{Z}$ and the methods for performing processing kinetics were recently described in detail in Zareen et al. (2005) and will therefore only be briefly outlined here. The fruit fly tRNase $\mathrm{Z}$ was designed to express from an internal methionine (M24, the first four encoded residues being MAAT) that is believed to be residue 1 of the nuclear form (Dubrovsky et al. 2004). Motif I mutations were constructed using the unique natural SacI site and the constructed XhoI site at the vector/insert boundary just beyond the stop codon, as previously described in Zareen et al. (2005). Because the SacI subcloning site (nt 1529) falls within the sequence that encodes the PxKxRN loop, these mutations were constructed using unique natural internal PflmI and Bpu10I sites (at nt 1146 and 1864, respectively), producing a 718-bp amplified DNA segment for subcloning. All variant DNA constructs were confirmed by sequencing (Herbert Irving Comprehensive Cancer Center, Columbia College of Physicians and Surgeons).

\section{Templates for T7 RNA polymerase transcription}

Runoff transcription with T7 RNA polymerase using plasmid templates has been described before; tRNA ${ }^{\text {His }}$ with a 36-nt $3^{\prime}$-end trailer and mature tRNA ${ }^{\text {His }}$-CCA were designed to run off using DraI and NsiI RE ends, respectively, for use as tRNase Z substrates (Levinger et al. 1995; Mohan et al. 1999). The new template for tRNA $^{\text {His }}$-AUG (a positive control for tRNase $\mathrm{Z}$ reaction designed to closely match the length of mature tRNA ${ }^{\mathrm{His}}$ ) was prepared by introducing a SacI site at the appropriate position by amplification using a mismatched 3 '-end primer and a universal 5 '-end primer. $\mathrm{tRNA}^{\mathrm{His}}$ with a long $3^{\prime}$-end trailer was either internally labeled during T7 transciption or unlabeled. Unlabeled tRNA ${ }^{\text {His }}$ CCA and -AUG were transcribed and gel-purified, and a small portion of each transcript was $5^{\prime}$ end labeled by dephosphorylation using shrimp alkaline phosphatase followed by treatment with polynucleotide kinase and $\gamma^{32} \mathrm{P}$-ATP and gel-purified again.

The $3^{\prime}$-AUG substrate appears to be $1 \mathrm{nt}$ longer than the $3^{\prime}$-CCA substrate (Fig. 6A,B), probably due to runoff transcription of cloned templates using different RE ends (-AUG: SacI and -CCA: Nsi I, respectively). T7 RNA polymerase is prone to incorporate an unencoded nucleotide at the $3^{\prime}$ end (Milligan et al. 1987), perhaps depending on the choice of runoff RE and the encoded $3{ }^{\prime}$-terminal sequence.

\section{tRNase Z processing kinetics}

Processing efficiencies were determined using labeled pre-tRNA ${ }^{\text {His }}$ with a 36-nt $3^{\prime}$-end trailer at a concentration of $\sim 10^{-10} \mathrm{M}$, more than 2 orders of magnitude lower than the lowest $\mathrm{k}_{\mathrm{M}}$ observed, so as to approximate zero-order kinetics, in which the \% product/ minute of reaction $(\mathrm{V} /[\mathrm{S}])$ evaluated early in a reaction time course is approximately proportional to V. For kinetic experiments, the concentration of labeled substrate was constant and total concentration of substrate was established by adding unlabeled pre-tRNA ${ }^{\text {His }}$. While \% product/minute (V/[S]) decreases with increasing [S], $\mathrm{V}$ (obtained by multiplying $\mathrm{V} /[\mathrm{S}]$ by $[\mathrm{S}]$ ) increases with $[S]$. The concentration of enzyme was adjusted as necessary based on results of efficiency experiments performed without added unlabeled substrate, and the substrate concentration range was adjusted so as to put the $\mathrm{k}_{\mathrm{M}}$ in the middle of the [S] range for each tRNase $\mathrm{Z}$ variant. Reliable, reproducible kinetic parameters were obtained with the lowest possible concentration of each variant enzyme. All variant enzymes were used for at least two kinetic experiments, and the reaction kinetics with the wildtype enzyme was performed alongside the variants every time a kinetic experiment was done. Comparisons to determine relative $\mathrm{k}_{\mathrm{cat}} / \mathrm{k}_{\mathrm{M}}$ were made between variant and wild type performed on the same day. 


\section{Multiple sequence alignments}

Multiple sequence alignments were prepared using ClustalW and displayed using GeneDoc.

\section{Modeling the structure of the PxKxRN loop and Motif I in relation to Motif II}

The tRNase Z structure model (de la Sierra-Gallay et al. 2005; PDB ID 1Y44) was visualized using New Cartoon in VMD with basic residues colored blue, acidic residues red, and neutral polar residues green.

\section{ACKNOWLEDGMENTS}

We thank Angelo Rossi for help with display of structure models and Zbigniew Dominski (University of North Carolina-Chapel Hill) for helpful discussions. This project was supported by grants from the NIH (S06GM08153) and the PSC-CUNY.

Received January 3, 2006; accepted February 28, 2006.

\section{REFERENCES}

Aebi, M., Kirchner, G., Chen, J.Y., Vijayraghavan, U., Jacobson, A., Martin, N.C., and Abelson, J. 1990. Isolation of a temperaturesensitive mutant with an altered tRNA nucleotidyltransferase and cloning of the gene encoding tRNA nucleotidyltransferase in the yeast Saccharomyces cerevisiae. J. Biol. Chem. 265: 16216-16220.

Baillat, D., Hakimi, M.A., Naar, A.M., Shilatifard, A., Cooch, N., and Shiekhattar, R. 2005. Integrator, a multiprotein mediator of small nuclear RNA processing, associates with the C-terminal repeat of RNA polymerase II. Cell 123: 265-276.

Castaño, J.G., Tobian, J.A., and Zasloff, M. 1985. Purification and characterization of an endonuclease from Xenopus laevis ovaries which accurately processes the $3^{\prime}$ terminus of human pre-tRNAMet(i) (3' pre-tRNase). J. Biol. Chem. 260: 9002-9008.

Chen, J.Y. and Martin, N.C. 1988. Biosynthesis of tRNA in yeast mitochondria. An endonuclease is responsible for the $3^{\prime}$-processing of tRNA precursors. J. Biol. Chem. 263: 13677-13682.

de la Sierra-Gallay, I.L., Pellegrini, O., and Condon, C. 2005. Structural basis for substrate binding, cleavage and allostery in the tRNA maturase RNase Z. Nature 433: 657-661.

Dominski, Z., Yang, X.C., and Marzluff, W.F. 2005a. The polyadenylation factor CPSF-73 is involved in histone-pre-mRNA processing. Cell 123: 37-48.

Dominski, Z., Yang, X.C., Purdy, M., Wagner, E.J., and Marzluff, W.F. 2005b. A CPSF-73 homologue is required for cell cycle progression but not cell growth and interacts with a protein having features of CPSF-100. Mol. Cell. Biol. 25: 1489-1500.

Dubrovsky, E.B., Dubrovskaya, V.A., Levinger, L., Schiffer, S., and Marchfelder, A. 2004. Drosophila RNase Z processes mitochondrial and nuclear pre-tRNA 3' ends in vivo. Nucleic Acids Res. 32: 255-262.

Frendewey, D., Dingermann, T., Cooley, L., and Söll, D. 1985. Processing of precursor tRNAs in Drosophila. Processing of the $3^{\prime}$ end involves an endonucleolytic cleavage and occurs after $5^{\prime}$ end maturation. J. Biol. Chem. 260: 449-454.

Ishii, R., Minagawa, A., Takaku, H., Takagi, M., Nashimoto, M., and Yokoyama, S. 2005. Crystal structure of the tRNA 3' processing endoribonuclease tRNase $\mathrm{Z}$ from Thermotoga maritima. J. Biol. Chem. 280: 14138-14144.

Kostelecky, B., Pohl, E., Vogel, A., Schilling, O., and MeyerKlaucke, W. 2006. The crystal structure of the zinc phosphodiesterase from Escherichia coli provides insight into function and cooperativity of tRNase Z family proteins. J. Bacteriol. 188: 16071614.
Kühn-Hölsken, E., Lenz, C., Sander, B., Luhrmann, R., and Urlaub, H. 2005. Complete MALDI-ToF MS analysis of cross-linked peptideRNA oligonucleotides derived from nonlabeled UV-irradiated ribonucleoprotein particles. RNA 11: 1915-1930.

Levinger, L., Vasisht, V., Greene, V., Bourne, R., Birk, A., and Kolla, S. 1995. Sequence and structure requirements for Drosophila tRNA $5^{\prime}$ and $3^{\prime}$ end processing. J. Biol. Chem. 270: 18903-18909.

Levinger, L., Bourne, R., Kolla, S., Cylin, E., Russell, K., Wang, X., and Mohan, A. 1998. Matrices of paired substitutions show the effects of tRNA D/T loop sequence on Drosophila RNase P and 3' -tRNase processing. J. Biol. Chem. 273: 1015-1025.

Levinger, L., Mörl, M., and Florentz, C. 2004a. A pathogenesisassociated mutation in human mitochondrial tRNALeu(UUR) leads to reduced $3^{\prime}$-end processing and CCA addition. J. Mol. Biol. 337: 535-544.

- 2004b. Mitochondrial tRNA $3^{\prime}$ end metabolism and human disease. Nucleic Acids Res. 32: 5430-5441.

Li, Z. and Deutscher, M.P. 1996. Maturation pathways for E. coli tRNA precursors: A random multienzyme process in vivo. Cell 86: $503-512$.

2002. RNase E plays an essential role in the maturation of Escherichia coli tRNA precursors. RNA 8: 97-109.

Marchfelder, A., Schuster, W., and Brennicke, A. 1990. In vitro processing of mitochondrial and plastid derived tRNA precursors in a plant mitochondrial extract. Nucleic Acids Res. 18: 1401-1406.

Marchfelder, A., Brennicke, A., and Binder, S. 1996. RNA editing is required for efficient excision of $\mathrm{tRNA}^{\mathrm{Phe}}$ from precursors in plant mitochondria. J. Biol. Chem. 271: 1898-1903.

McClain, W.H., Guerrier-Takada, C., and Altman, S. 1987. Model substrates for an RNA enzyme. Science 238: 527-530.

Milligan, J.F., Groebe, D.R., Witherell, G.W., and Uhlenbeck, O.C. 1987. Oligoribonucleotide synthesis using T7 RNA polymerase and synthetic DNA templates. Nucleic Acids Res. 15: 87838798.

Minagawa, A., Takaku, H., Takagi, M., and Nashimoto, M. 2004. A novel endonucleolytic mechanism to generate the CCA $3^{\prime}$ termini of tRNA molecules in Thermotoga maritima. J. Biol. Chem. 279: $15688-15697$.

Mohan, A. and Levinger, L. 2000. The effects of matrices of paired substitutions in mid-acceptor stem on Drosophila tRNA ${ }^{\mathrm{His}}$ structure and end-processing. J. Mol. Biol. 303: 605-616.

Mohan, A., Whyte, S., Wang, X., Nashimoto, M., and Levinger, L. 1999. The $3^{\prime}$ end CCA of mature tRNA is an anti-determinant for eukaryotic 3'-tRNase. RNA 5: 245-256.

Mörl, M. and Marchfelder, A. 2001. The final cut. The importance of tRNA 3'-processing. EMBO Rep. 2: 17-20.

Nashimoto, M. 1992. Characterization of the spermidine-dependent, sequence-specific endoribonuclease that requires transfer RNA for its activity. Nucleic Acids Res. 20: 3737-3742.

- 1997. Distribution of both length and $5^{\prime}$ terminal nucleotides of mammalian pre-tRNA $3^{\prime}$ trailers reflect properties of $3^{\prime}$ processing endoribonuclease. Nucleic Acids Res. 25: 1148-1154.

Ow, M.C. and Kushner, S.R. 2002. Initiation of tRNA maturation by RNase E is essential for cell viability in E. coli. Genes \& Dev. 16: 1102-1115.

Pellegrini, O., Nezzar, J., Marchfelder, A., Putzer, H., and Condon, C. 2003. Endonucleolytic processing of CCA-less tRNA precursors by RNase Z in Bacillus subtilis. EMBO J. 22: 4534-4543.

Ryan, K., Calvo, O., and Manley, J.L. 2004. Evidence that polyadenylation factor CPSF-73 is the mRNA $3^{\prime}$ processing endonuclease. RNA 10: 565-573.

Schiffer, S., Rosch, S., and Marchfelder, A. 2002. Assigning a function to a conserved group of proteins: The tRNA $3^{\prime}$-processing enzymes. EMBO J. 21: 2769-2777.

. 2003. Recombinant RNase $Z$ does not recognize CCA as part of the tRNA and its cleavage efficiency is influenced by acceptor stem length. Biol. Chem. 384: 333-342.

Schilling, O., Späth, B., Kostelecky, B., Marchfelder, A., MeyerKlaucke, W., and Vogel, A. 2005. Exosite modules guide substrate 
recognition in the ZiPD/ElaC protein family. J. Biol. Chem. 280: 17857-17862.

Shi, P.Y., Weiner, A.M., and Maizels, N. 1998. A top-half tDNA minihelix is a good substrate for the eubacterial CCA-adding enzyme. RNA 4: 276-284.

Spath, B., Kirchner, S., Vogel, A., Schubert, S., Meinlschmidt, P., Aymanns, S., Nezzar, J., and Marchfelder, A. 2005. Analysis of the functional modules of the tRNA $3^{\prime}$ endonuclease (tRNase Z). J. Biol. Chem. 280: 35440-35447.

Takaku, H., Minagawa, A., Takagi, M., and Nashimoto, M. 2003. A candidate prostate cancer susceptibility gene encodes tRNA 3' processing endoribonuclease. Nucleic Acids Res. 31: 2272-2278.

Tavtigian, S.V., Simard, J., Teng, D.H.F., Abtin, V., Baumgard, M., Beck, A., Camp, N.J., Carillo, A.R., Chen, Y., Dayananth, P., et al. 2001. A candidate prostate cancer susceptibility gene at chromosome 17p. Nat. Genet. 27: 172-180.
Vogel, A., Schilling, O., Spath, B., and Marchfelder, A. 2005. The tRNase Z family of proteins: Physiological functions, substrate specificity and structural properties. Biol. Chem. 386: 1253-1264.

Xiao, S., Scott, F., Fierke, C.A., and Engelke, D.R. 2002. Eukaryotic ribonuclease P: A plurality of ribonucleoprotein enzymes. Annu. Rev. Biochem. 71: 165-189.

Yan, H., Zareen, N., and Levinger, L. 2006. Naturally occurring mutations in human mitochondrial pre-tRNASer(UCN) can affect the tRNase $\mathrm{Z}$ cleavage site, processing kinetics and substrate secondary structure. J. Biol. Chem. 281: 3926-3935.

Zareen, N., Yan, H., Hopkinson, A., and Levinger, L. 2005. Residues in the conserved His domain of fruit fly tRNase $\mathrm{Z}$ that function in catalysis are not involved in substrate recognition or binding. J. Mol. Biol. 350: 189-199.

Zhu, L. and Deutscher, M.P. 1987. tRNA nucleotidyltransferase is not essential for Escherichia coli viability. EMBO J. 6: 2473-2477. 

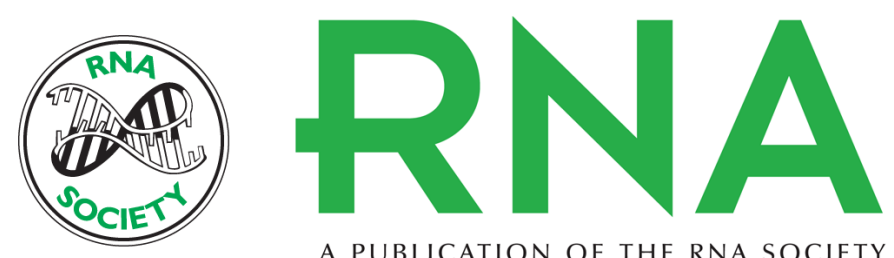

A PUBLICATION OF THE RNA SOCIETY

\section{Residues in two homology blocks on the amino side of the tRNase Z His domain contribute unexpectedly to pre-tRNA 3 ' end processing}

Neela Zareen, Angela Hopkinson and Louis Levinger

RNA 2006 12: 1104-1115

References This article cites 43 articles, 23 of which can be accessed free at:

http://rnajournal.cshlp.org/content/12/6/1104.full.html\#ref-list-1

License

Email Alerting Receive free email alerts when new articles cite this article - sign up in the box at the Service top right corner of the article or click here.

To subscribe to RNA go to:

http://rnajournal.cshlp.org/subscriptions 